\title{
Essais
}

ESSAIS

Revue interdisciplinaire d'Humanités

\section{Le camp d'internement des Milles : enjeux mémoriels (1939-2013)}

\section{Cécile Denis}

\section{(2) OpenEdition}

1 Journals

Édition électronique

URL : http://journals.openedition.org/essais/5682

DOI : $10.4000 /$ essais.5682

ISSN : 2276-0970

Éditeur

École doctorale Montaigne Humanités

\section{Édition imprimée}

Date de publication : 15 janvier 2015

Pagination : 70-92

ISBN : 978-2-9544269-4-5

ISSN : 2417-4211

\section{Référence électronique}

Cécile Denis, « Le camp d'internement des Milles : enjeux mémoriels (1939-2013) », Essais [En ligne], 6 | 2015, mis en ligne le 30 novembre 2020, consulté le 10 décembre 2020. URL : http://

journals.openedition.org/essais/5682 ; DOI : https://doi.org/10.4000/essais.5682 


\section{Le camp d'internement des Milles : enjeux mémoriels (1939-2013)}

\section{Cécile Denis}

Dans un article publié en 2007 et dédié aux lieux de migration et de mémoire franco-allemands, le germaniste Thomas Keller et l'historien JeanMarie Guillon évoquent " l'éclosion problématique des initiatives visant à créer des lieux et des jours de mémoire $»^{1}$. Deux ans plus tard, une gradation s'opère lorsque les historiens Pascal Blanchard et Isabelle Veyrat-Masson tentent de comprendre "pourquoi et comment des guerres de mémoire existent, s'affirment, se transforment et contribuent in fine à "l'identité nationale" "2. Or, c'est précisément entre les années 2006 et 2009, au moment où ces questions se posent avec acuité, que le projet d'inaugurer le mémorial du camp d'internement des Milles est finalisé au terme d'une longue et difficile période de gestation. Le site occupe les bâtiments et le terrain d'une ancienne tuilerie située près d'Aix-en-Provence. Le bâtiment principal représentant $25000 \mathrm{~m}^{2}$ de surface habitable a été de 1939 à 1942 le camp d'internement et de déportation le plus important du Sud-Est de la France. Contrairement à beaucoup d'autres camps construits ex nibilo dans un but précis et détruits lorsqu'ils n’avaient plus lieu d'être, le camp des Milles a repris son activité initiale de briqueterie entre les années 1946 et 2003. À l'heure actuelle, il s'agit du seul camp d'internement français dont le bâtiment est encore intact et accessible au public. Le mémorial du Camp des Milles a ouvert ses portes en septembre 2012. En février 2013, le Mémorial de la Shoah lui a consacré un volet dans son exposition sur les nouveaux " musées - mémoriaux » de la Shoah en France ${ }^{3}$, aux côtés d'autres lieux de mémoire tels qu'Yzieux, Drancy et Rivesaltes. Mais ces trois camps

1 Thomas Keller, Jean-Marie Guillon, «Introduction. Du lieu de migration au lieu de commémoration - du lieu de commémoration au lieu de migration ", in Thomas Keller, JeanMarie Guillon (éd.) Lieux de migrations/lieux de mémoire franco-allemands, Cahiers d'Études Germaniques, n 53, 2007, p. 7.

2 Pascal Blanchard et Isabelle Veyrat-Masson, "Les guerres de mémoires : un objet d'étude ? ", in Tracés 3/2009 (n HS-9), p. 43-52.

3 Exposition au Mémorial de la Shoah "Les nouveaux musées-mémoriaux de la Shoah en France " du 16 septembre 2012 au 31 mars 2013. 
d'internement ne peuvent être présentés au sein d'une même exposition que parce qu'ils sont considérés sous un angle particulier en tant que projets architecturaux et scénographiques transmettant la mémoire des lieux de transit. Pour le reste de leur histoire, il apparaît difficile d'attribuer un statut identique aux Milles, à Rivesaltes et à Drancy. Afin de distinguer les particularités du cas des Milles, il convient de rappeler quelques chiffres ainsi que trois étapes dans son histoire qui sont préalables à la question du " transit ». Les Milles compte parmi les 200 camps dans lesquels 600000 personnes ont été incarcérées entre 1939 et 1946, selon les estimations de l'historien Denis Peschanski, ${ }^{4}$ spécialiste de l'internement français. Les premiers camps d'internement sont majoritairement installés dans le Sud-Ouest pour " accueillir " les réfugiés espagnols. Le camp des Milles n'est pas concerné par cette première étape, il correspond à la deuxième : il n'est pas ouvert au début de l'année 1939 mais en septembre de la même année, après la déclaration de guerre à l'Allemagne, afin d'interner les ressortissants des puissances ennemies en attente d'un visa pour émigrer. La troisième étape est celle de l'emprisonnement des personnes que l'on désigne alors communément par le terme générique "d'indésirables " après la défaite militaire française en mai 1940 : les Allemands et les «ex-Autrichiens » se réclamant du droit d'asile, les ressortissants d'Europe centrale qui sont originaires d'anciens territoires germaniques ou austro-hongrois, mais aussi les communistes après le pacte germano-soviétique et leurs " sympathisants » tels que les sociaux-démocrates ou les anarchistes, ou encore les francs-maçons et les tziganes. La circulaire ministérielle en date du 17 septembre $1939^{5}$ regroupe les camps d'internement en trois catégories : le camp des Milles se classe dans les " camps de rassemblement des étrangers " destinés aux éléments " tranquilles ». Les deux autres classifications comprennent les camps dits "semi-répressifs " pour les personnes "à surveiller ", Gurs par exemple, ou les camps répressifs comme Le Vernet. Un décret-loi du 12 novembre 1938, élargi par la loi du 18 novembre 1939, permet l'internement "sur décision du préfet » des « individus dangereux pour la défense nationale ou la sécurité publique $»^{6}$. Ces individus sont alors internés

4 Denis Peschanski, Les camps français d'internement, 1938-1946, thèse d'État sous la direction d'Antoine Prost, Paris, 2000, p. 1. Ces estimations concernent la période qui s'étend de 1939, date de la fondation de Rieucros en Ariège, à 1946, fermeture des Alliers en Charente. Le premier camp a servi à interner les réfugiés espagnols avant de devenir rapidement un camp d'internement réservé aux femmes. Le second était un camp d'internement destiné aux " nomades" (Roms, Tziganes).

5 «Circulaire du ministère de l'Intérieur du 17 septembre 1939 sur les étrangers et les apatrides suspects, dangereux ou indésirables, et sur les mesures d'internement les concernant reproduite ", in Jacques Grandjonc, Theresia Grundtner (éd.), Zone d'ombres 1933-1944, exil et internement d'Allemands et d'Autrichiens dans le Sud de la France, Aix-en-Provence, Éditions ALINEA et ERCA, 1990, p. 204-207.

6 « Décret relatif aux mesures à prendre à l'égard des individus dangereux pour la défense nationale ou la sécurité publique ", 18 novembre 1939, reproduit in Robert Mencherini, « De la galaxie des Milles aux rafles de Juifs en Provence ", in Robert Mencherini (éd.), Provence- 
non pas parce qu'ils ont commis un délit mais parce qu'ils représentent un danger potentiel, fussent-ils d'authentiques antinazis. À partir de 1941, certains camps tels que Rivesaltes ou les Milles changent de fonction et se transforment en camps de transit pour les victimes " raciales " du régime national-socialiste. Ils deviennent donc les antichambres d'Auschwitz et font alors partie intégrante du programme d'extermination " raciale ». Leur population change, les femmes et les enfants, jusqu'alors internés séparément, sont rassemblés pour partir dans des convois qui ont pour destination Drancy. Mais contrairement au camp de Drancy, situé en zone Nord et réquisitionné par l'armée allemande dès juin 1940 pour l'internement des prisonniers de guerre puis pour la déportation des Juifs, les camps d'internement sont majoritairement situés en zone Sud, zone non-occupée jusqu'en novembre 1942. Par conséquent, ils sont avant tout au cour du régime de Vichy, et ne sont pas l'instrument privilégié de l'occupant pour sa politique de répression. Alors que la plupart de ces camps sont situés dans le Sud-Ouest comme c'est le cas pour Gurs, Saint Cyprien, Le Vernet ou Argelès, Les Milles se distingue au sein de cette nébuleuse : il est le principal camp du Sud-Est ${ }^{7}$ avec plus de 10000 personnes internées dont 2000 Juifs qui sont déportés entre août et septembre 1942. À titre de comparaison, Rivesaltes, le plus grand camp du Sud-Ouest en nombre de détenus, a accueilli 21000 personnes en deux ans. 2300 d'entre elles auraient rejoint Drancy. Mais la spécificité des Milles tient surtout à la composition de la population internée. Barbara Vormeier estime que 12000 à 14000 Allemands et environ 5000 Autrichiens auraient été concernés par les mesures d'internement en France ${ }^{8}$. De 1941 à 1942, aux Milles, environ un millier de prisonniers en moyenne sont originaires du Reich allemand?

Comme on le sait, la France a mis des décennies avant de se pencher sur l'histoire de Vichy et les camps d'internement français, qui ont fait l'objet d'un oubli ou d'une indifférence collectifs. Après soixante-dix ans de désintéressement à l'égard de son histoire, nous pouvons nous demander pour quelles raisons le passé du camp des Milles a resurgi et dans quelles mesures son historiographie s'inscrit dans un phénomène mémoriel plus vaste concernant les lieux de mémoire français du régime de Vichy. Comme le résume Henry Rousso, le postulat des historiens de la mémoire est en effet que "la relation au passé connaît des évolutions et qu' elle possède une historicité propre

Auschwitz, de l'internement des étrangers à la déportation des Juifs (1939-1944), Aix-en-Provence, Publications de l'Université de Provence, 2007, p. 311.

7 Pierre Laborie, "Vichy et l'exclusion : un miroir impitoyable ", in Monique-Lise Cohen, Éric Malo, Les camps du Sud-Ouest de la France, 1939-1944, Toulouse, Privat, 1994, p. 18.

8 Barbara Vormeier, "La situation des réfugiés en provenance d'Allemagne (septembre 1939 juillet 1942) », in Jacques Grandjonc, Theresia Grundtner (éd.), op. cit., p. 192.

9 Robert Mencherini, op. cit., p. 21. 
qu'il s'agit de mettre à jour $»^{10}$. Nous utiliserons le terme de "phénomène mémoriel ${ }^{11}$ pour désigner de façon neutre les différents événements qui ont marqué l'histoire du mémorial des Milles et qui introduisent ou concluent des périodes historiques puis historiographiques. Notre analyse se déroulera en trois temps : nous allons tout d'abord nous intéresser à la fonction remplie par ce lieu, de sa création en 1882 en tant que tuilerie jusqu'en 2003, date de la cessation de l'activité industrielle. Nous nous pencherons ensuite sur les étapes et les difficultés posées par le retour de la mémoire, principalement à partir des années 1980. Enfin, nous analyserons de quelle façon le musée actuel présente les différentes phases de l'histoire du camp à proprement parler, ainsi que les répercussions qu'a eues la révélation de cette mémoire sur le plan local et les ambitions que porte le mémorial pour son avenir.

\section{Le site des Milles et ses différentes fonctions (1882-2009)}

Le 10 septembre 2012, à l'occasion de l'inauguration du « Site-Mémorial », le président de la Fondation du Camp des Milles Alain Chouraqui, directeur de recherches au CNRS, s'interrogeait : comment passe-t-on de "l'ordinaire de la tuilerie des Milles à l'extraordinaire d'Auschwitz » ${ }^{12}$ ? La Société des Tuileries de la Méditerranée est créée le 23 mai 1882 par Édouard Rastoin. La tuilerie des Milles est construite la même année à six kilomètres d'Aix-en-Provence, en plein cour de la Provence, dans une zone rurale où elle dispose de la matière première (argiles de la rive droite de l'Arc au lieu-dit les Tuilières) et à proximité de l'agglomération marseillaise. De plus, le site industriel bénéficie d'un point d'eau, la rivière l'Arc, et surtout d'une ligne de chemin de fer qui relie Paris, Lyon et Marseille. Après plusieurs décennies de bon fonctionnement, la crise de 1929 conduit à des difficultés d'ordre social, technique et économique, et l'usine ferme ses portes en 1937. Mais les atouts géographiques, les infrastructures et les voies de communication, qui avaient contribué à l'installation et au bon fonctionnement de l'entreprise, vont s'avérer être des avantages pour réutiliser le site, cette fois-ci en tant que camp d'internement.

10 Henry Rousso, "Les dilemmes d'une mémoire européenne ", in Christian Delacroix, François Dosse, Patrick Garcia (éd.), Historicités, La Découverte, 2009, p. 207.

11 Cette notion est à distinguer du concept de «paradigme mémoriel » défini par Hélène Camarade comme un "système dominant d'interprétations et de représentations du passé à un moment donné, qui s'appuie sur un consensus et qui est appelé à être remplacé par un autre paradigme. " Cf. "La mémoire du national-socialisme en République fédérale d'Allemagne, essai de périodisation ", in Hélène Camarade, Elizabeth Guilhamon, Claire Kaiser (éd.), Le national-socialisme dans le cinéma allemand contemporain, Villeneuve d'Ascq, Presses Universitaires du Septentrion, 2013. Avec le terme de "phénomène mémoriel ", nous désignons ici les manifestations d'un paradigme mémoriel à l'échelle locale.

12 Alain Chouraqui, intervention du 10 septembre 2012 à l'inauguration du "Site-Mémorial " (archives personnelles de l'auteur). 
Les trois étapes du camp d'internement de 1939 à 1942 et ses changements de fonction de 1942 à 1946

Louverture du camp des Milles a lieu peu après la déclaration de guerre à l'Allemagne. Le 4 septembre 1939, les locaux désaffectés de la briqueterie sont réquisitionnés par le gouvernement français et rouvrent leurs portes sous commandement militaire. Dès la mi-septembre, un nombre exceptionnellement élevé de personnalités ayant fui le nazisme est emprisonné. Si cette nouvelle disposition s'applique à tous les camps, elle a de grandes conséquences aux Milles en raison du nombre élevé d'opposants allemands et autrichiens au national-socialisme présents dans la région. La présence d'intellectuels et d'artistes germanophones au camp des Milles peut paraître étonnante de prime abord mais elle trouve son explication dans une histoire antérieure, celle de l'exil. L'établissement d'Allemands et d'Autrichiens sur la Côte d'Azur remonte au début du XX $\mathrm{XX}^{\mathrm{e}}$ siècle. Des peintres allemands partent à la recherche de la lumière vers la Provence. Ils s'installent dans les petits ports au bord de la Méditerranée. Lorsque les premiers émigrés quittent l'Allemagne en 1933, c'est tout naturellement qu'ils font appel à leurs amis et forment des communautés littéraires et artistiques en exil. Marseille occupe également une position stratégique. Sous condition d'obtention d'un visa, le port méditerranéen permet de quitter la France par bateau. Et pour ceux qui ne parviennent pas à obtenir le sésame, Marseille constitue alors une étape, généralement après Paris et Lyon ou encore Nice, dans leur fuite vers l'Espagne. Aux Milles, il n'est donc pas étonnant que les nationalités les plus représentées soient les Allemands et les " ex-Autrichiens " : en 1941 et 1942, les chiffres oscillent entre 450 et 756 Allemands et entre 159 et 304 Autrichiens environ, alors que le camp compte 829 internés en mars 1941. De janvier à juin 1942, on dénombre entre 1300 et 1400 internés $^{13}$. Ces décomptes ne concernent que les hommes. Les internés poursuivent leur activité malgré les circonstances. Tous les domaines sont concernés : la peinture et le dessin (Max Ernst, Robert Liebknecht), la littérature (Golo Mann, Alfred Kantorowicz, Lion Feuchtwanger, Franz Hessel), les sciences (Otto Meyerhof) pour ne citer que les plus célèbres détenus. Ainsi, les archives du camp des Milles se caractérisent par une production artistique exceptionnellement riche et diversifiée (plus de 300 œuvres actuellement recensées) qui s'étend de 1939, date de l'ouverture, jusqu'à l'été 1942, moment où le camp perd sa fonction première et devient un camp de transit. Des cours ou des conférences sont donnés, on écrit puis on interprète des pièces de théâtre. Les internés se retrouvent au premier niveau de la briqueterie, dans les anciens fours Hoffmann utilisés auparavant pour la cuisson des briques, pour travailler ou y installer

13 Robert Mencherini, op. cit., p. 21. 
leur salle de spectacle, appelée "Katakombe ». Les murs sont ornés d'une multitude de peintures murales à la taille et aux motifs variés (masques de Comedia dell'arte, fleurs...) Parfois des commandes officielles sont passées par les autorités : d'imposantes peintures murales sont effectuées dans le réfectoire des gardiens, une petite salle située dans la cour de la briqueterie, en $1941^{14}$. Les moissons et les vendanges, Le Banquet des Nations, Rêve de nourriture et Le cortège des prestataires sont quatre fresques mettant en scène des banquets et des cornes d'abondance tandis que les prisonniers sont affamés. Les œuvres réalisées empruntent à plusieurs styles, dont le constructivisme, et sont à attribuer à plusieurs auteurs, l'un d'entre eux étant vraisemblablement Karl Boldek. Lors de cette première phase de l'histoire du camp, des "Commissions permanentes de criblage " décident le maintien de l'internement ou la libération, et le nombre de détenus s'amenuise. C'est ainsi par exemple qu'Alfred Kantorowicz est libéré et saisit l'occasion pour émigrer aux États-Unis via Marseille. En revanche, l'écrivain Lion Feuchtwanger regagne sa demeure de Sanary. Entre les mois d'avril et de juin 1940, on comptabilise en tout 300 internés qui quittent les Milles et sont provisoirement transférés à Lambesc, petit village situé au nord d'Aix-en-Provence ${ }^{15}$.

La deuxième phase intervient après la défaite militaire française et l'invasion du territoire. Les étrangers en attente d'un visa et qui avaient été libérés grâce aux commissions de criblage sont de nouveau emprisonnés aux Milles, et les mesures d'internement à leur égard sont durcies. Le journal local, Le Petit-Var, publie le 21 mai 1940 l'ordre donné par le général commandant la $\mathrm{XV}^{\mathrm{e}}$ Région : "Les ressortissants allemands et étrangers de nationalité indéterminée, mais d'origine allemande [...] devront sans délai rejoindre le centre de rassemblement du camp des Milles ». Au moment de la signature de l'armistice ${ }^{16}$, le 22 juin 1940, le camp des Milles est placé sous l'autorité du ministère de l'Intérieur. Certains, comme Lion Feuchtwanger, sont internés pour la deuxième fois. Son roman autobiographique, intitulé Le diable en France (Der Teufel in Frankreich) ${ }^{17}$, relate le quotidien des prisonniers et l'absurdité de leur internement. Le livre n'a pas été achevé par l'auteur lui-même, il s'interrompt

14 Cf. brochure Site-Mémorial du camp des Milles, Aix-en-Provence, Fondation du Camp des Milles, année inconnue, p. 15, multigraphié.

15 Claire Lutrin-Le Pors, Lambesc : la mémoire oubliée, lieu d'édition inconnu, Éditions Tourne la page, 2012, $48 \mathrm{p}$.

16 Extrait de l'article 19 de la convention d'armistice du 22 juin 1940 : «Le Gouvernement français est tenu de livrer sur demande tous les ressortissants allemands désignés par le Gouvernement du Reich et qui se trouvent en France. " "Convention d'armistice signée à Rethondes le 22 juin 1940 ", en ligne sur la Digithèque des matériaux juridiques et politiques de l'Université de Perpignan : http://mjp.univ-perp.fr/france/1940armistice.htm [consulté le 14/01/2013].

17 Lion Feuchtwanger, Unholdes Frankreich, Mexico, El Libro Libre, 1942, 330 p. Cet ouvrage est plus connu sous le titre Der Teufel in Frankreich. 
juste avant son évasion. C'est son épouse Martha qui rédige le dernier chapitre et raconte leurs fuites respectives vers les États-Unis. Voici comment l'auteur décrit la population incarcérée aux Milles en 1940 :

Les ordres d'internement étaient appliqués à la lettre ; les autorités subalternes avaient manifestement reçu la consigne d'interner plutôt trop de gens que trop peu. On ne s'en tenait pas aux seuls Allemands, Autrichiens et Tchèques ainsi que le stipulait le décret, car on trouvait également parmi nous des Luxembourgeois, des Hollandais, des Belges et des Scandinaves. Il était impossible de faire l'appel. Une fois entré dans le camp, on ne pouvait plus en sortir, même s'il s'agissait de toute évidence d'un excès de zèle ou de l'erreur d'un policier.

C'était grotesque de voir le genre de personnes qu'on avait rassemblées là sous prétexte qu'elles avaient pu entretenir des rapports avec la Cinquième Colonne. [...] L'incarcération de tant de gens qui avaient montré de manière irréfutable leur farouche opposition au nazisme constituait une stupide et déplorable

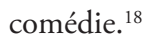

À cette population hétérogène s'ajoute une nouvelle catégorie d'internés car le décret du 4 octobre 1940 stipule que les Juifs étrangers doivent à leur tour être internés. C'est ainsi que 6500 Juifs expulsés du Palatinat, du Bade et du Wurtemberg ${ }^{19}$ sont d'abord dirigés vers le camp d'internement de Gurs dans le cadre de l'action Bürckel-Wagner ${ }^{20}$. Certains d'entre eux seront par la suite transférés au camp des Milles.

Les femmes sont quant à elles transférées dans des centres d'internement pour femmes. En juin 1940, à Marseille, 150 ressortissantes allemandes et autrichiennes sont les premières internées à l'hôtel Bompard. Les hôtels Terminus des ports et du Levant sont également réquisitionnés pour l'occasion $^{21}$. Comme c'est le cas pour les hommes au camp des Milles, les personnes ressortissantes du Reich sont les plus nombreuses : dans le cas des femmes, leur nombre dépasse les $60 \%$ parmi les internées ${ }^{22}$.

18 Lion Feuchtwanger, Le Diable en France, Paris, Éditions Jean-Cyrille Godefroy, 1985, p. 38.

19 Barbara Vormeier, "La situation des réfugiés en provenance d'Allemagne (septembre 1939 juillet 1942) ", in Jacques Grandjonc, Theresia Grundtner, op. cit., p. 197.

20 "À la fin de l'été 1940, le RSHA a mis au point un projet colossal : profiter de la défaite française pour installer dans une lointaine colonie de la France, l'île de Madagascar, une sorte de gigantesque ghetto dans lequel seraient rassemblés les Juifs raflés. [...] Pour en hâter la réalisation, il convient donc de passer immédiatement à l'exécution de la première phase du plan. L'opération Bürckel" constitue justement l'occasion de tester la bonne volonté de l'administration française tout en impliquant davantage le régime de Vichy dans une forme de collaboration directe. Rappelons en outre qu'en octobre 1940 il n'est plus seulement question d'expulsion des Juifs, et qu'il n'est pas encore question de "solution final" ». Cf. Claude Laharie, "La déportation et l'internement au camp de Gurs des 6538 Juifs allemands originaires du pays de Bade et de Palatinat (19401943) », in Monique-Lise Cohen, Éric Malo, op. cit., p. 107.

21 Sylvie Orsoni, «Étrangères indésirables : les centres d'internement féminin à Marseille (19401942) ", in Robert Mencherini, op. cit., p. 39.

22 Sylvie Orsoni, op. cit., p. 45. 
Enfin, un tournant se produit en juin 1942. Theodor Dannecker, chef de la section de la Gestapo chargée de la " question juive " et représentant d'Adolf Eichmann en France, demande la déportation de 40000 Juifs de France. Le programme doit être réalisé en trois mois et prévoit une déportation par secteurs géographiques : 15000 en provenance de la zone occupée, $15000 \mathrm{du}$ Grand Paris et plusieurs milliers de la zone non-occupée ${ }^{23}$. Le 16 juin 1942, René Bousquet, secrétaire général de la police de Vichy, accepte de livrer 10000 Juifs de la zone Sud ${ }^{24}$. En août et septembre 1942, les Milles devient par conséquent un camp de déportation vers Auschwitz via Drancy ou Rivesaltes. 2000 personnes environ, hommes, femmes et enfants, sont déportées en trois semaines. Le 15 septembre, la zone Sud toute entière a livré 10000 Juifs. En octobre, ce chiffre monte à 10500 Juifs déportés ${ }^{25}$. L'administration de Vichy a alors atteint les quotas réclamés par l'Allemagne et le camp redevient un centre de transit pendant quelques mois, jusqu'en décembre 1942.

La zone non-occupée est envahie par l'armée allemande en novembre 1942. Le bâtiment du camp est réquisitionné pour le stockage des munitions et passe sous le contrôle militaire allemand. À partir de la deuxième quinzaine du mois d'août 1944, au moment du débarquement allié en Provence, l'armée américaine y cantonne durant un an. Jusqu'en 1946, le site est probablement délaissé.

\section{Reprise de l'activité économique de 1946 à 2009}

Dès l'année 1946 et jusqu'en 1987, l'activité de briqueterie reprend sous la direction de la famille Rastoin. Le management familial devient alors régional puis multinational. Après la succession de différents groupes, la société Lafarge, groupe français de matériaux de construction, acquiert le site en 1987. En fonction durant plusieurs années, l'ancienne tuilerie fait ensuite office d'entrepôt et cesse définitivement toute activité en 2003. Cela permet de conserver le site, bâtiment et réfectoire, sans modification majeure même si certaines des œuvres peintes dans les fours par les artistes sont effacées lorsque ceux-ci sont rallumés : l'indifférence règne au niveau local en ce qui concerne le camp d'internement et son histoire. Ce passé n'est plus évoqué jusqu'à la fin des années 1970. Qui sont donc les instigateurs du retour de cette mémoire? Toutes les phases où la tuilerie a servi de camp d'internement sont-elles considérées avec le même intérêt ou a-t-on privilégié une mémoire en particulier ?

23 Serge Klarsfeld, « La livraison par Vichy des Juifs de zone libre dans les plans SS de déportation des Juifs de France ", in Monique-Lise Cohen, Éric Malo, op. cit., p. 140.

24 Sylvie Orsoni, op. cit., p. 44.

25 Serge Klarfeld, op. cit., p. 151. 


\section{La préservation du site et le retour de la mémoire : étapes et instigateurs (1942-2012)}

Afin de saisir les spécificités du retour de la mémoire dans le cas du camp des Milles, il convient de rappeler les principales étapes qui constituent l'historiographie du régime de Vichy et des camps d'internement. Au lendemain de la Seconde Guerre mondiale, les gouvernements de la IV puis de la $V^{e}$ République instituent ce que l'historien Henry Rousso nomme le "résistancialisme ${ }^{26}$, un mythe selon lequel les Français auraient unanimement et naturellement résisté depuis le début de la Seconde Guerre mondiale. Le gouvernement de Vichy est perçu comme une parenthèse dans l'histoire de France et la question de la collaboration des autorités françaises avec le gouvernement hitlérien devient un tabou national. Ce mythe unificateur a pour objectif de restaurer et de défendre une certaine cohésion de la nation française, et contribue de la sorte à la reconstruction du pays aux sens propre et figuré. La briqueterie des Milles s'inscrit parfaitement dans cette phase. Les anciens surveillants affectés au camp reprennent le cours de leur vie, ils ne livrent aucun récit. La population locale est indifférente à cette histoire : à ses yeux, elle n'est pas concernée puisque l'internement touchait l'« antiFrance ${ }^{27}$. Par conséquent, elle n’identifie pas ce site comme un lieu de migra$\operatorname{tion}^{28}$ traumatisant. Seules quelques personnes dans la population locale ont acquis bien plus tard le titre de " Juste parmi les nations » pour avoir aidé des prisonniers juifs. Mais vraisemblablement confrontés à l'indifférence locale et en prises aux soucis du quotidien (et certainement mus aussi par la discrétion), ils n'ont parlé que bien plus tard de leurs actes. Les porteurs de souvenir, et donc de mémoire, sont les anciens internés alors dispersés, mais ceux-ci ont quitté ce lieu où ils ont juste passé quelques mois.

\section{Les germanistes de l'université d'Aix-en-Provence et la mémoire des intellectuels allemands}

À partir de 1942, seuls de rares témoignages sur le camp voient le jour, le plus célèbre étant le livre de Lion Feuchtwanger intitulé Le Diable en France ${ }^{29}$.

26 Henry Rousso, Le Syndrome de Vichy de 1944 à nos jours, Paris, Seuil, 2e éd., 1990, 414 p. L'édition originale date de 1987.

27 Sous le régime de Vichy, on entend par là les individus à qui l'on attribue la défaite de 1940, à savoir les Juifs, les communistes et les francs-maçons.

28 Le " lieu de migration " désigne un endroit géographique où les migrants ne sont pas nés et n'ont pas grandi. Ils doivent se l'approprier, en apprenant la langue par exemple. Dans leurs récits postérieurs, le décalage entre leur culture et celle du lieu d'accueil a toujours une place très importante. Cf. Thomas Keller, Jean-Marie Guillon, op. cit., p. 9.

29 On peut citer comme premiers témoignages les ouvrages suivants : Henri Manen, Au fond de l'abîme, (journal du camp des Milles), lieu d'édition inconnu, Éditions Ampelos, 2013, 
Ainsi, dès les années 1950, le lecteur allemand peut apprendre l'existence du camp des Milles bien avant que cette histoire ne soit découverte par les Français. Il n'est donc pas étonnant que l'histoire du camp des Milles éveille, dans un premier temps, l'intérêt des germanistes aixois qui, lorsqu'ils en apprennent l'existence trente ans après les premiers témoignages, mettent à l'honneur les célébrités allemandes internées dans ce site.

Les recherches des germanistes s'inscrivent dans un courant historiographique plus vaste, d'échelle internationale, communément appelé la révolution paxtonienne. Peu après la mort du général De Gaulle en 1970, des initiatives issues de pays étrangers, telles que le documentaire franco-suisse $L e$ Chagrin et la Pitié de Max Ophüls en 1971 et l'ouvrage pionnier de l'historien américain Robert Paxton La France de Vichy en 1973, mais aussi l'ouverture progressive des archives apportent une nouvelle perspective et inaugurent un cycle de remise en cause des mémoires convenues.

La recouvrance de la mémoire des Milles débute en 1973, à Berlin-Est. À cette époque, la " division ${ }^{30}$ de la mémoire est encore vivace dans les deux États allemands : l'Allemagne de l'Ouest met encore principalement à l'honneur les formes de résistance chrétienne ou humanitaire, comme celle de la Rose Blanche, ou bien la résistance militaire. L'Allemagne de l'Est, de son côté, a construit son identité en se définissant comme un État antifasciste, or le fascisme est, selon l'approche marxiste, une conséquence du capitalisme. Ce sont donc les résistants communistes qui incarnent pendant de longues années l'unique forme de résistance tolérée dans la mémoire officielle de la RDA. Nous l'avons mentionné plus haut, les mesures d'internement en France ont concerné les Allemands et les communistes. Après la guerre, bon nombre d'anciens internés allemands communistes s'établissent en RDA et y entretiennent la mémoire des Allemands en France, une fois que ce sujet est

56 p. Des extraits du journal ont été envoyés à des pasteurs à Nîmes et à Genève, traduits en allemand et publiés dans une revue juive new-yorkaise Aufbau/Reconstruction, $\mathrm{n}^{\circ}$ 51, le 18 décembre 1942. Le titre prête cependant à confusion : «Ich habe es gesehen; Erster Bericht aus den Deportations-Tagen in Gurs. Aus dem Tagebuch eines französischen Geistlichen » ("Je l'ai vu. Premier compte-rendu des journées de déportation à Gurs. Extrait du journal intime d'un ecclésiastique "). Or, il est bien question des Milles et non pas de Gurs. Cf. Henri Manen, " au fond de l'abîme ", in Jacques Grandjonc, Theresia Grundtner, op. cit., p. 353-354. Des extraits traduits en allemand sont également disponibles depuis les années 1990 dans l'ouvrage d'Edwin Maria Landau, Lager in Frankreich: Überlebende und ihre Freunde: Zeugnisse der Emigration, Internierung und Deportation, Augsburg, Brandt, 1991, 259 p. L'autre ouvrage est le roman autobiographique de Lion Feuchtwanger paru en 1942 lors de son exil aux ÉtatsUnis, op. cit. La première traduction française date vraisemblablement de 1985, Paris, Éditions Jean-Cyrille Godefroy, 1985, 227 p.

30 Hélène Camarade, "Les Allemands dans la résistance en France (1940-1945) : un trou de mémoire en République fédérale d'Allemagne ", in Cahiers d'Études Germaniques, n 57, Marseille, Université de Provence, 2009, p. 143. 
homologué par la mémoire officielle à partir des années $1970^{31}$. C'est à cette époque qu'un germaniste aixois, Jacques Grandjonc, professeur à l'Université de Provence, spécialiste de linguistique allemande, prend connaissance de l'histoire des Milles en rencontrant Karl Obermann, un collègue historien, membre du parti communiste est-allemand, le SED, et ancien interné32.

En 1977, le germaniste Gilbert Badia, professeur à l'Université de Paris VIII, membre du Parti Communiste français, connu pour ses amitiés est-allemandes, initie les premières recherches sur l'exil allemand antinazi en France ${ }^{33}$. C'est dans ce contexte marqué en RDA par un début de travail de mémoire sur la présence de communistes allemands en France pendant la guerre et, en France, par un début d'approche critique de l'histoire de Vichy et sur l'exil allemand que les premières recherches scientifiques d'André Fontaine, élève de Jacques Grandjonc, débutent deux ans plus tard sur le camp des Milles. Une exposition est organisée à Aix-en-Provence en 1981 au sujet des œuvres d'art produites aux Milles et un article d'André Fontaine paraît dans les Cahiers d'Études Germaniques la même année ${ }^{34}$, mais la connaissance du site ne semble pas dépasser un cercle restreint d'universitaires. Cette première étape du retour de la mémoire met à l'honneur une seule mémoire, celle des artistes germanophones, comme l'explique rétrospectivement Alain Chouraqui, actuel président de la Fondation du Camp des Milles :

31 Hélène Camarade, «La mémoire du national-socialisme en République fédérale d'Allemagne, essai de périodisation ", op. cit.

32 Karl Obermann (1905-1987) est membre de la «Freie Sozialistische Jugend » en 1925 puis de la "Sozialistische Arbeiterpartei Deutschlands » en 1931. Il immigre en France en 1933 où il suit des cours d'histoire à la Sorbonne. En 1936, il adhère au parti communiste allemand (KPD). En 1939, il est emprisonné dans les camps d'internement français et émigre aux États-Unis en 1941. En 1946, il s'installe en zone d'occupation soviétique en passant par l'URSS. Il soutient sa thèse d'histoire en 1950 et devient professeur à la Humboldt-Universität de Berlin. De 1956 à 1960, il dirige l'Institut pour l'histoire allemande à la nouvelle Académie des Sciences de Berlin (Institut für Deutsche Geschichte der Deutschen Akademie der Wissenschaften zu Berlin). Membre du SED, il représente la première génération d'historiens marxistes-léninistes en RDA. Cf. Mario Keßler, "Zurück aus amerikanischem Exil, der Remigrant und Historiker Karl Obermann", Universität Potsdam, Wissenschaft Aktuell, mai 2010. Article en ligne : http://www.uni-potsdam.de/u/putz/mai00/oberm.htm [consulté le 07/10/2013].

33 Parmi les principaux résultats de recherche de ce groupe, on peut citer les trois ouvrages suivants : Les Barbelés de l'exil : études sur l'émigration allemande et autrichienne (1938-1940), Grenoble, Presses universitaires de Grenoble, 1979 ; Exilés en France : souvenirs d'antifascistes allemands émigrés (1933-1945), Paris, Maspero, 1982 ; Les bannis de Hitler, Paris, Presses universitaires de Vincennes, 1984.

34 André Fontaine, "Le camp des Milles (septembre 1939 - mars 1943) Historique provisoire ", in Cahiers d'Études Germaniques, n 5, Marseille, Université de Provence, 1981, p. 287. 
Mais les peintures et la célébrité de beaucoup des internés ont longtemps conduit à concentrer l'attention sur cette part de "culture allemande en France " - au point que fut évoqué le projet de faire des Milles une annexe du Musée des Beaux-arts d'Aix ! La période où le camp servit à l'internement des étrangers masqua ainsi souvent l'importance de la dernière période, celle de la déportation anonyme des Juifs. ${ }^{35}$

Ce premier et modeste réveil de la mémoire constitue cependant un phénomène mémoriel précis : le passage du lieu de migration, c'est-à-dire celui d'une expérience personnelle et individuelle, à un lieu de souvenir (en allemand "Erinnerungsort "), un site " qui, à travers des souvenirs isolés, reprend vie spontanément $»^{36}$.

\section{Enjeux liés à la préservation du site}

Le véritable tournant pour la préservation de la mémoire a lieu en 1982. Lorsqu'on annonce la destruction prochaine du réfectoire des gardiens qui est orné de peintures murales, la mémoire latente du camp des Milles resurgit. Deux hypothèses nous ont été livrées sur le déroulement des faits qu'il apparaît difficile de vérifier. Un premier récit raconte que ce serait le sous-préfet qui aurait informé une association d'anciens résistants et déportés de la destruction prochaine de la salle des peintures. Ceux-ci se seraient alors mobilisés avec le Conseil représentatif des institutions juives de France (CRIF) et la mairie d'Aix-en-Provence. Une action est entreprise auprès de la Direction régionale des affaires culturelles. Une autre hypothèse provient de l'article de Jacques Grandjonc et de Philippe Joutard ${ }^{37}$, où il est fait mention que le "directeur de l'équipe de recherches " (vraisemblablement le même Jacques Grandjonc, mais le nom n'est pas cité) serait intervenu auprès de Jack Lang pour inscrire la salle des peintures à l'inventaire supplémentaire des monuments historiques. Selon cette deuxième hypothèse, le CRIF, les organisations d'anciens internés et déportés et des personnalités politiques - dont les noms demeurent inconnus malgré nos recherches - ne seraient intervenus qu'après cela pour transformer le réfectoire en musée.

Une instance de classement est prise le 5 novembre 1982 par le ministère de la Culture afin de protéger cette partie du site. Le réfectoire est ensuite inscrit à l'inventaire supplémentaire des monuments historiques le 3 novembre 1983. Conséquence du "sauvetage " de la salle des peintures, un "Comité de coordination pour la sauvegarde du Camp des Milles » et un "Musée mémorial de la Déportation, de la Résistance et de l'Internement " sont constitués dès

35 Alain Chouraqui, "Le camp des Milles ou la mémoire au forceps ", in l'Arche, $\mathrm{n}^{\circ}$ 460, Paris, mars 1996, p. 68.

36 Thomas Keller, Jean-Marie Guillon, op. cit., p. 9-10.

37 Jacques Grandjonc, Philippe Joutard, op. cit., p. 20, note de bas de page $\mathrm{n}^{\circ} 25$. 
1985 sous l'égide de Denise Toros Marter, déportée à Auschwitz à 16 ans, Sidney Chouraqui, engagé volontaire dans les Forces Françaises Libres, et Louis Monguilan, résistant déporté à Mauthausen. Dans une conférence donnée au Mémorial de la Shoah le 22 mai 2013, Denis Peschanski a insisté sur « l'importance des initiatives prises par la société civile comme les autorités régionales pour la préservation des sites lieux de mémoire $»^{38}$. Dans le cas des Milles, nous assistons ainsi dès le début des années 1980 à une mobilisation de bénévoles. Ce sont ces exhortations qui ont engendré un début de prise de responsabilité par les pouvoirs publics. Dix années plus tard, en novembre 1993, la salle des peintures est classée monument historique et sa gestion confiée au ministère des anciens combattants et victimes de guerre.

\section{Les ouvrages scientifiques des années 1990}

Parmi les premiers ouvrages scientifiques prétendant à l'exhaustivité figure le livre d'André Fontaine intitulé Un camp de concentration à Aix-en-Provence? Le Camp d'étrangers des Milles, 1939-194339. Il est cependant contesté dans un article par Jacques Grandjonc et Philippe Joutard ${ }^{40}$ qui dénoncent son manque de rigueur scientifique ${ }^{41}$. En 1990 paraît le livre Zone d'ombres sous la direction de Jacques Grandjonc et Theresia Grundtner, qui porte avant tout sur les migrations des Allemands et des Autrichiens et dans lequel le camp des Milles n'est évoqué qu'en marge. Néanmoins, l'avant-propos inscrit ces recherches dans un phénomène mémoriel et un courant de recherche plus vastes :

Au moment où croît dans le public l'intérêt pour un aspect occulté de notre histoire nationale et européenne - l'exil et l'internement d'étrangers réfugiés sur le sol français, puis leur émigration outre-mer ou leur participation à la Résistance, ou encore leur déportation depuis les camps du Midi - nous avons décidé de doubler les résultats déjà assurés avec ceux que nous a permis d'établir la recherche poursuivie au cours des dernières années. ${ }^{42}$

38 Denis Peschanksi, «Les camps d'internement dans le Sud de la France en 1939 », conférence tenue le 22 mai 2013 à Paris au Musée d'Art et d'Histoire du Judaïsme.

39 André Fontaine, Un camp de concentration à Aix en Provence? Le Camp d'étrangers des Milles, 1939-1943, Cahors, Édisud, 1989, 245 p.

40 Philippe Joutard fait actuellement partie du conseil d'administration de la Fondation du camp des Milles, Mémoire et Éducation, dans la partie "Collège des personnalités qualifiées ".

41 Jacques Grandjonc, Philippe Joutard, "La perversion du travail d'historien, ou comment ne pas écrire l'histoire de l'internement en France pendant la guerre. ", in Cahiers d'études germaniques, $\mathrm{n}^{\circ} 17$, Aix-en-Provence, automne 1989, p. 9-20. Cet article analyse le travail précité et fait ressortir plusieurs éléments qui remettent en question la rigueur scientifique de l'ouvrage : des imprécisions concernant les chiffres ou les dates, ou encore l'énumération superflue de détails physiques et de la vie privée de plusieurs personnalités liées au camp des Milles.

42 Jacques Grandjonc, Theresia Grundtner, op. cit., p. 7. 
Il faut cependant attendre 1999 pour que le premier ouvrage scientifique de référence paraisse, celui de Doris Obschernitzki, ${ }^{43}$ Letzte Hoffnung-Ausreise: Die Ziegelei von Les Milles 1939-1942: Vom Lager für unerwünschte Ausländer zum Deportationszentrum [Dernier espoir : l'émigration. La briqueterie des Milles de 1939 à 1942 : du camp pour les étrangers indésirables au centre de déportation $]^{44}$. Nous avons cité le cas du livre de Feuchtwanger plus haut : encore une fois, le lecteur germanophone accède à des informations inédites ou peu connues en France, ce livre n'ayant à ce jour jamais été traduit.

\section{Concurrence des mémoires}

Si les premières publications scientifiques caractérisent la décennie des années 1990, c'est également à cette période que l'on voit naître un foisonnement d'initiatives éparses pour recouvrer les mémoires du camp des Milles. À l'image de la présidence du Comité de coordination pour la sauvegarde du Camp des Milles - composée, comme nous le disions précédemment, de Denise Toros Marter, déportée à Auschwitz, de Sidney Chouraqui, ancien des Forces Françaises Libres et de Louis Monguilan, résistant déporté à Mauthausen - les différentes mémoires de la déportation, de la Shoah et de la résistance sont honorées et coexistent. On constate également que ces mémoires sont portées par des personnes qui ne sont pas d'anciens internés, ces derniers étant en revanche sollicités par les chercheurs en Allemagne, par Doris Obschernitzki par exemple. Parmi les Français qui encouragent le devoir de mémoire aux Milles, chacun trouve tout naturellement dans ce site un écho à son histoire personnelle et les objectifs different selon les centre d'intérêt de chacun et le groupe qu'il représente : les déportés pour raisons raciales, les résistants ou bien encore les soldats des Forces Libres.

43 Doris Obschernitzki, née en 1946 au Danemark, est germaniste et historienne, elle a soutenu sa thèse sur le roman de Franz Kafka, Le Château, en 1978, sous la direction de Walter Höllerer. Au cours d'un séjour d'une dizaine d'années en France, à Aix-en-Provence, elle a été associée aux recherches de Jacques Grandjonc et de son équipe à l'université de Provence. Cf. Doris Obschernizki, Frankreichs deutsche Emigranten, Texte und Erinnerungen an Jacques Grandjonc 1933-2000, Berlin, Hentrich \& Hentrich, 2003, p. 275. [Les émigrés allemands de la France, textes et souvenirs à propos de Jacques Grandjonc, 1933-2000].

44 Doris Obscherniztki, Letzte Hoffnung-Ausreise: Die Ziegelei von Les Milles 1939-1942: Vom Lager für unerwünschte Auslander zum Deportationszentrum, Berlin, Hentrich \& Hentrich, 1999, $454 \mathrm{p}$. 
Ces différentes initiatives marquent cependant le passage du lieu d'émigration, c'est-à-dire celui d'une expérience personnelle et individuelle, à un lieu de mémoire (en allemand "Gedächtnisort ") caractérisé par le début d'écriture et donc de fictionnalisation : en d'autres termes, le lieu mémorisé est mis en scène dans des ouvres (textes, expositions, films ${ }^{45} \ldots$ ) $^{46}$.

Dans ce contexte, trois événements marquants ont lieu mais sans lien entre eux : le Conseil d'Administration de l'Association Philatélique du Pays d'Aix, dont le président est Yvon Romero, décide d'acquérir un lot d'une dizaine de lettres écrites par des internés au camp des Milles, retrouvées par hasard. L'objectif est avant tout une forme de préservation du patrimoine. Au même moment, Yad Vashem décerne le titre de Juste des Nations à plusieurs personnes qui ont sauvé des Juifs au camp des Milles. Quelques distinctions avaient déjà été accordées dès les années 1960 et 1980, notamment au pasteur Donadille et à Auguste Boyer, mais leur nombre augmente dans les années 1990. Nous pouvons citer les cas de Cyrille Argenti, d'Edmond et de Nelly Bartoloni ou du couple Donnier. La troisième initiative a lieu en 1992, année marquée par la plainte déposée contre la SNCF pour crime contre l'humanité. Dans ce contexte, les anciens wagons deviennent le symbole de la déportation des Juifs et c'est vraisemblablement pour cette raison qu'un " wagon-souvenir " est installé par l'Amicale des anciens déportés d'Auschwitz en novembre de la même année à l'endroit même où les départs avaient lieu. Son inauguration, à la date anniversaire des pogroms de la " Nuit de cristal " et pour commémorer les cinquante ans des déportations en zone Sud, est l'occasion d'une vaste opération éducative et culturelle à Aix et à Marseille, intitulée « Mémoire pour demain ». Le travail du premier comité est relayé par l'Association du Wagon-Souvenir et du Site-Mémorial du Camp des Milles dont Denise Toros Marter, Sidney Chouraqui et Louis Monguilan deviennent les co-présidents. Cette dernière initiative marque une étape supplémentaire dans le travail de mémoire, à savoir la volonté, commune aux trois co-présidents, de transformer le lieu de mémoire en lieu de commémoration, et elle s'inscrit dans une démarche d'ampleur nationale : une initiative similaire a lieu au Vernet deux ans plus tard. Peu à peu, ces interventions contribuent à changer les mentalités en faisant accepter la réalité de Vichy à un large public. En 1995, Jacques Chirac "apure les contentieux mémoriels ${ }^{47}$ grâce à son discours du 16 juillet. Prononcé lors des commémorations de la rafle du Vel' d'Hiv', le chef de l'État reconnaît la responsabilité de l'État français dans les

45 Les Milles, le train de la liberté. Ce film de Sébastien Grall réalisé en 1995 relate l'épisode du " train fantôme ». Lorsque l'arrivée des troupes nazies est annoncée en mai 1940, le commandant du camp affrète un train pour évacuer secrètement les prisonniers.

46 Thomas Keller, Jean-Marie Guillon, op. cit., p. 9-10.

47 Olivier Wieviorka, La Mémoire désunie. Le souvenir politique des années sombres, de la Libération à nos jours, Paris, Éditions du Seuil, coll. "L'Univers historique ", 2010, 303 p. 
déportations et son discours ne suscite pas de polémique, ce qui couronne un changement de paradigme mémoriel au sein de la société française. En l'an 2000, la soutenance, puis la publication de la thèse d'État de Denis Peschanski sur les camps d'internement, qui complète l'ouvrage d'Anne Grynberg ${ }^{48}$ paru l'année précédente, confirment qu'une étape a en effet été franchie dans ce processus mémoriel.

Certes, les efforts pour que les différentes mémoires coexistent et soient honorées sans esprit de concurrence entre elles sont louables. Mais il manque d'une part une cohérence d'ensemble et d'autre part un lien plus clair avec l'histoire du site. Ses spécificités et les anciens internés sont en effet absents de ces démarches. Nous pourrions nous approprier ici l'analyse que Tzvetan Todorov livre dans son ouvrage Les abus de la mémoire ${ }^{49}$ : le fait de conserver toutes les mémoires sans distinction et dans les moindres détails ne constitue pas encore un travail de mémoire. En effet, un travail de mémoire doit obligatoirement procéder à une sélection pour mettre en lumière les éléments importants et donc la spécificité de chaque mémoire. Dans le cas des Milles, on assiste dans les années 1990 à une situation paradoxale en ce qui concerne les phénomènes mémoriels : il existe à la fois une indifférence ou une méconnaissance locale, un réveil des différentes mémoires qui se fait sans distinction grâce à des associations de bénévoles, et les premières productions scientifiques de qualité sont produites en langue allemande ou diffusées en français au sein des groupes de germanistes.

\section{Le tournant des années 2000}

Lors de sa conférence donnée au Mémorial de la Shoah en 2013, Denis Peschanski a évoqué le début d'une nouvelle phase muséographique $e^{50}$ dans les années 1990. Celle-ci serait marquée par la prise de conscience selon laquelle un musée doit, pour fonctionner, être de taille importante et qu'il a besoin d'une légitimité scientifique. Cette étape est franchie lorsque Sydney Chouraqui passe le flambeau à son fils, Alain Chouraqui, directeur de recherche au CNRS. À partir de ce moment-là, le président de la Fondation n'aura de cesse de donner une cohérence et un fondement scientifique aux différentes initiatives nées dans les années 1990 sans pour autant privilégier une mémoire en particulier. La mémoire n’est plus aux mains des victimes

48 Anne Grynberg, Les camps de la honte, les internés juifs des camps français 1939-1944, Paris, Édition La Découverte, 2000.

49 Tzvetan Todorov, Les abus de la mémoire, Paris, Seuil, 1998, p. 14.

50 Camille Mazé, "Le musée comme trace historique. Un lieu de redéfinition permanent des rapports à "l'identité" entre science et politique ", dans ce même numéro. Camille Mazé fait notamment référence au passage des " musées de la nation » aux " musées de l'Europe » observé depuis la fin des années 1980 . 
ou des témoins et ce changement générationnel marque le passage à la quatrième étape du schéma proposé par Thomas Keller et Jean-Marie Guillon que l'on peut résumer ainsi : le lieu de migration (première étape) devient un lieu de souvenir (deuxième étape), puis un lieu de mémoire (troisième étape) et, enfin un lieu de commémoration. Cette quatrième étape est atteinte lorsque la politique de mémoire "relie la conscience collective au lieu et le distingue ${ }^{51}$. Cette évolution n'aurait sans doute pas eu lieu sans la création de la Fondation pour la mémoire de la Shoah en l'an 2000, comme nous le verrons plus tard.

L'avenir de la briqueterie et du site tout entier se décide peu de temps après, en 2002, d'une part avec la naissance d'un comité de pilotage présidé par Alain Chouraqui, à la suite d'une " déclaration commune sur un projet de lieu de mémoire " signée par la mairie d'Aix-en-Provence, la société Lafarge et l'association du wagon-souvenir, et d'autre part avec une structure juridique porteuse du projet, l'association Mémoire du camp des Milles. En 2004, c'est l'ensemble du site (sept hectares) qui est inscrit comme Monument historique par le ministère de la Culture. Mais la construction du mémorial est alors freinée par des problèmes de financement, malgré les études sur le potentiel de fréquentation. Finalement, la Fondation pour la mémoire de la Shoah achète le site au groupe Lafarge et confie sa gestion à l'association Mémoire du camp des Milles afin de réaliser un site mémoriel. La première pierre est posée en 2009 sous le parrainage du président de la République. Cette même année, la "Fondation du Camp des Milles - Mémoire et Éducation » est créée. Le 10 septembre 2012, le "Site-Mémorial » est inauguré et ouvert au public en présence du Premier ministre Jean-Marc Ayrault.

\section{Le mémorial du camp des Milles aujourd'hui : de la " mémoire révérence à la mémoire référence ${ }^{52}$}

\section{Les acteurs actuels de la mémoire et le statut du «Site-Mémorial »}

Le mémorial comprend actuellement le site dans son ensemble : l'ancienne tuilerie où le musée a été installé, la salle des peintures et le wagon-souvenir. Comme nous l'avons précisé plus haut, cette salle des peintures, ou « réfectoire des gardiens", est classée monument historique depuis 1993. Lancienne tuilerie a été, quant à elle, inscrite comme Monument historique en 2004. Cela signifie que les statuts de la tuilerie et de la salle sont différents : un bâtiment classé ne peut être transformé ou cédé sans que l'on en informe le ministre et une partie

51 Thomas Keller, Jean-Marie Guillon, op. cit., p. 9.

52 Alain Chouraqui, "Faire vivre un haut lieu de mémoire et d'éducation ", in brochure Sitemémorial du camp des Milles, op. cit., p. 25. 
de l'entretien est financé par l'État. Cette inégalité de statut peut également expliquer que les différentes parties du site n'aient pas jusque-là bénéficié du même traitement mémoriel. À cela s'ajoutent des questions d'ordre pratique qui ont longtemps conduit à privilégier certains lieux en particulier tels que la salle des peintures et le wagon-souvenir, plus petits en surface et moins coûteux pour l'entretien. À l'heure actuelle, la sauvegarde, l'ouverture au public des bâtiments du camp ainsi que son aménagement relèvent de la " Fondation du camp des Milles - Mémoire et Éducation », ce qui garantit une homogénéité dans le traitement des différentes parties du site. Établissement reconnu d'utilité publique, c'est donc un organisme régi par le droit privé. Il est dirigé par un large conseil d'administration : les partenaires publics (l'État et les collectivités locales), associatifs (Association Mémoire du camp d'Aix les Milles, Fondation pour la Mémoire de la Shoah...) et privés (les entreprises mécènes telles qu'Orange et la Caisse d'Épargne). Un Conseil scientifique, international et pluridisciplinaire, composé d'universitaires, est responsable des contenus du "Site-Mémorial ». Parmi ses membres, on peut citer des professeurs d'histoire tels qu'Anne Grynberg, JeanMarie Guillon, Robert Mencherini, Philippe Mioche. Sont également présents des sociologues (Chantal Borde-Benayoun, Angelika Gausmann), philosophes (Georges Bensoussan), un conservateur du patrimoine (François Gasnault), Serge Klarsfeld, Jacqueline Ursch (Directrice des Archives Départementales des Bouches-du-Rhône), et Olivier Lalieu (responsable de l'aménagement des lieux de mémoire et des projets externes du Mémorial de la Shoah et rapporteur du Conseil Scientifique $)^{53}$.

\section{Le parcours muséographique}

La fonction du mémorial est étroitement liée à l'organisation de l'espace. Le parcours muséographique s'effectue en trois temps qui représentent les différents thèmes de réflexion voulus par la Fondation. Dans les trois cas, la pédagogie s'adresse en priorité à un jeune public dans un souci évidemment culturel mais aussi d'éducation citoyenne. Le premier volet est historique. Il s'agit de présenter le contexte international et national puis l'histoire régionale avant de s'intéresser aux différentes étapes de l'histoire du camp ${ }^{54}$.

53 Cette liste ne prétend pas à l'exhaustivité.

54 Ici, il faut souligner que les initiatives nées dans les années 1990 sont mises à contribution. Par exemple, les recherches menées par les philatélistes Yvon Romero et Guy Marchot. Touchés par le contenu des lettres qu'ils avaient trouvées, ces derniers s'intéressent au parcours des expéditeurs et des destinataires, avant et après-guerre, et rencontrent parfois leurs descendants. Considérées comme de nouvelles sources d'archives, ces lettres constituent un témoignage exceptionnel sur la vie des internés et des militaires. Les résultats sont parus en juin 2012 dans un ouvrage qui clôture leur travail et précède en même temps l'inauguration du mémorial. Cf. Guy Marchot, Lettres des internés du camp des Milles, Chirat Saint Just-la-Pendue, Éditions Association Philatélique du Pays d'Aix, 2012. 
Concrètement, le visiteur entre dans l'ancienne tuilerie mais ne la reconnaît pas : les premières pièces traversées ont été rénovées et réaménagées. Elles donnent à voir une exposition " traditionnelle » et un équipement moderne avec la projection de films, des écrans interactifs. Des bornes audiovisuelles reconstituent les destins individuels d'internés. Le deuxième volet est mémoriel : le visiteur ne quitte pas le bâtiment mais en passant d'une pièce à l'autre, il sort des salles du musée pour entrer dans le lieu de mémoire à proprement parler puisqu’il parcourt les salles d'internement et qu'il peut observer les traces laissées par les détenus. Les dispositifs muséographiques sont alors très discrets, à dessein. Quelques panneaux indiquent la fonction des espaces et l'emplacement de graffiti effectués par les détenus mais l'information fournie en première partie suffit au visiteur pour donner un sens aux espaces parcourus. L'objectif est ici de ressentir la force évocatrice de ces lieux restaurés par des archéologues mais volontairement laissés en l'état. Des escaliers conduisent à un étage et le visiteur entre alors dans le troisième volet, l'aspect réflexif. D'une pièce à l'autre, il quitte les anciennes salles pour entrer de nouveau dans un musée moderne qui n'est pas sans rappeler la première partie du musée. Mais cette fois-ci, il ne s'agit plus de reconstituer l'histoire du lieu. Des connaissances pluridisciplinaires sont présentées afin de mieux comprendre les engrenages qui conduisent à l'intolérance, le basculement de la démocratie à l'autoritarisme, la responsabilité individuelle et collective face au crime de masse. Cette étape est en quelque sorte une station expérimentale dans laquelle le visiteur établit un parallèle avec les expériences du passé pour se livrer à une réflexion sur le présent. Ici encore, il faut souligner l'exceptionnel équipement informatique mis à disposition qui sollicite la réflexion personnelle (films, archives sonores, présentation de résultats scientifiques, dispositifs interactifs notamment au moyen de casques ou d'écrans tactiles) et qui permet de répondre presque sur mesure aux questions de chacun. Le visiteur accède ensuite aux espaces extérieurs du camp, dont la salle des peintures murales et la cour de la briqueterie. La visite se termine en empruntant le "Chemin des Déportés " qui conduit au Wagon du Souvenir, ancien lieu des départs en déportation. Afin de compléter ce parcours muséographique, la Fondation propose également un centre de ressources qui s'étend sur $400 \mathrm{~m}^{2}$ et contient environ 5000 documents de nature diverse (des livres, mais également des films ou encore des bandes dessinées ainsi que des archives). De nombreuses activités culturelles sont également organisées dans différentes salles de projection ${ }^{55}$. Le retour d'une culture vivante dans le Site-Mémorial est considéré comme un hommage à la mémoire des internés qui ont résisté à la persécution par la création.

55 Des conférences, par exemple sur « Etty Hillesum, la résistance par l'esprit " le 9 novembre 2013, des concerts, notamment le 8 juillet 2013 avec le quatuor Bella qui interprète de la musique de chambre, des prestations théâtrales ou artistiques, des opéras, par exemple l'opéra pour enfants Brundibár de Hans Krása, interprété les 21 et 22 juin 2013. 
Ainsi, en plusieurs étapes, en alternant la grande histoire et les petites histoires, le visiteur prend non seulement connaissance et conscience du passé mais il est également invité à se livrer à une réflexion, et ses émotions sont également sollicitées. De cette manière, la Fondation allie lieu de mémoire, d'hommage et espace éducatif ouvert à un large public, sans pour autant privilégier une mémoire en particulier.

\section{Répercussions}

Le "Site-Mémorial » a pour ambition de devenir l'un des premiers équipements culturels français. Dans un article des Cahiers d'Études Germaniques datant de 2007, Alain Chouraqui et Olivier Lalieu écrivent : " Les résultats d'une étude universitaire des publics situent le potentiel de fréquentation de ce lieu au-delà de 100000 entrées annuelles, ce qui le placerait dans le premier groupe des équipements culturels français, et en tête pour Aix-Marseille " ${ }^{56}$.

Si une partie du financement du mémorial, établissement sans but lucratif, est issue de fonds privés tels qu'Alteor, spécialisé en immobilier, tourisme et équipement de la maison, Orange installations ou encore WhatMatters informatique, le fait que la région d'Aix-Marseille ait été élue capitale européenne de la culture 2013 a sans nul doute également joué un rôle. Le choix de cette région a été opéré en septembre 2008 et la Fondation du Camp des Milles a été, comme nous l'avons dit précédemment, reconnue d'utilité publique un an plus tard. Nous pouvons donc nous interroger sur la nature du lien qui unit ces deux événements : il s'agit vraisemblablement d'une opération de communication d'envergure visant d'une part à faire connaître le mémorial à un très large public et, de l'autre, permettant des retombées positives en termes d'image.

Cette publicité positive pour la région d'Aix-Marseille semble être la répercussion la plus évidente de la création du mémorial des Milles. Mais il serait réducteur de considérer le travail de la Fondation uniquement sous cet angle. La naissance du mémorial s'inscrit également dans un phénomène mémoriel de plus grande ampleur en contribuant à briser les tabous liés à l'histoire de Vichy. En effet, le mémorial constitue un soutien de poids pour la redécouverte à l'échelle locale de cet épisode de l'histoire de France, comme cela a été le cas pour la commune de Lambesc. Situé au nord d'Aix-en-Provence, ce village a temporairement remplacé le camp des Milles du mois d'avril au mois de juin 1940. Brièvement évoqué dans l'ouvrage d'André Fontaine ${ }^{57}$ et sur une carte du livre d'Anne Grynberg, Les camps de la honte $e^{58}$, il a attiré

56 Alain Chouraqui, Olivier Lalieu, "Le camp des Milles : une histoire plurielle, une mémoire pour demain. ", in Thomas Keller, Jean-Marie Guillon, op. cit., p. 124.

57 André Fontaine, op. cit.

58 Ibid. 
l'attention de Claire Lutrin-Le Pors, étudiante en histoire qui y était domiciliée. Après s'être heurtée à un silence généralisé, celle-ci a fini par découvrir une liste de 343 détenus aux archives départementales des Bouches-duRhône. Parallèlement à ces recherches, une association aixoise "Résister aujourd'hui " demande dès 1995 l'apposition d'une plaque sur le lieu de l'internement et se heurte au refus de la mairie. Il faut attendre des élections locales et le changement de municipalité pour que cela soit suivi d'effets au mois d'avril 2010. Trois ans plus tard, la municipalité de Lambesc, l'association précitée et Claire Lutrin-Le Pors publient un livret ${ }^{59}$ explicatif préfacé par Robert Mencherini, également membre du conseil scientifique du mémorial des Milles. Une rencontre de ces scientifiques et du maire avec la population est organisée conjointement avec l'exposition "Vichy en Provence " en juin 2013. Celle-ci est organisée dans le cadre d'une opération de médiation pédagogique et culturelle proposée par les archives départementales des Bouchesdu-Rhône. Créé en 2004, le "Semi des Archives" est un semi-remorque conçu comme espace itinérant d'exposition. Il a pour objet de valoriser les archives auprès du grand public et des établissements scolaires. Sur la brochure introductive de l'exposition est précisé que celle-ci « entre en résonance avec l'ouverture du Site-Mémorial du camp des Milles ».

Ainsi le lien est nettement marqué entre les deux phénomènes mémoriels, celui du camp de Lambesc, victime d'un déni, et celui du mémorial du camp des Milles en pleine phase d'expansion. Le mémorial devient de la sorte un outil mémoriel qui encourage les processus d'imputabilité chers à Paul Ricœur, ${ }^{60}$ et réalise ainsi son objectif majeur : devenir " un lieu témoin qui prend le relais des témoins".

\section{Conclusion}

La question de la périodisation de la mémoire a été posée en introduction. Nous constatons dans un premier temps que la mémoire du camp d'internement des Milles s'inscrit dans un paradigme mémoriel plus vaste : celui du rapport à l'histoire de Vichy et de son oubli. Dans le cas des Milles, celui-ci a la particularité d'être plus ou moins volontaire. Parce qu'il s'agit d'un ancien lieu de migration, la population locale a en effet du mal à s'approprier ce phénomène mémoriel perçu comme étranger. Puis cette mémoire en sommeil se réveille, particulièrement tôt dans notre cas, dès le début des

59 Claire Lutrin-Le Pors, op. cit.

60 "L'imputabilité constitue une capacité franchement morale. Un agent humain est tenu pour l'auteur véritable de ses actes, quelle que soit la force des causes organiques et physiques. Assumée par l'agent, elle le rend responsable, capable de s'attribuer une part des conséquences de l'action ; s'agissant d'un tort fait à autrui elle dispose à la réparation et à la sanction finale. " Paul Ricœur, «Devenir capable, être reconnu », in Esprit, n 7, Paris, juillet 2005, p. 126. 
années 1980. Ce phénomène particulier s'inscrit dans deux courants historiographiques plus vastes, celui de la relation au passé de Vichy et les recherches sur l'exil allemand en France. Confrontés au déni ou à l'oubli, les "passeurs de mémoire »-qui, nous le rappelons, ne sont pas les anciens internés et sont rarement en contact avec eux - ont apporté leur contribution en fonction de leur sensibilité, de leurs centres d'intérêts et de leurs moyens pour réhabiliter cette mémoire jusque-là latente. Ainsi, les différentes mémoires ont longtemps coexisté entre le réfectoire, dont les fresques murales rappellent les artistes allemands, et le wagon-souvenir, symbole des déportations mais aussi des actions des Justes. Nous assistons alors à un phénomène mémoriel caractérisé par le foisonnement des initiatives qui, si elles ont le mérite d'exister et de coexister sans se faire ombrage, manquent néanmoins de cohérence et sèment parfois une certaine confusion. Les années 2000 inaugurent un troisième phénomène mémoriel : celui de musées visant un public international et nécessitant, par conséquent, des infrastructures adaptées ainsi qu'un conseil scientifique de qualité. Pour preuve de son efficacité, le réseau scientifique international créé par la Fondation du Camp des Milles et l'Université d'Aix-Marseille a été reconnu comme «Chaire Unesco » en $2013^{61}$, ce qui implique l'obligation de produire un compte rendu biennal ${ }^{62}$. Cela apparaît comme la suite logique de la coopération renforcée mise en œuvre au moyen d'une convention entre la Fondation du Camp des Milles et Éducation et Aix-Marseille Université. L'impulsion a sans nul doute été apportée par Alain Chouraqui, qui n'a eu de cesse non seulement de préserver ce lieu de mémoire mais également d'en faire le vecteur d'un projet éducatif au moyen d'une démarche historique et scientifique. Aujourd'hui, le parcours muséographique allie l'information et la prévention, l'histoire et la didactique tout en restant ouvert et accessible à un large public. Dans son discours d'inauguration, Alain Chouraqui s'interrogeait : "Face à d'indécentes concurrences de mémoires, la démarche proposée ici ne peut-elle aussi aider à construire une solide convergence des mémoires, fondée sur ce qu'il y a de commun dans leur expérience des comportements humains, des pires aux plus admirables? "63

61 In site internet du Mémorial du camp des Milles, rubrique Fondation, objectifs : http://www. campdesmilles.org/fondation-objectifs.html [consulté le 29/08/2013].

62 In site internet du ministère des Affaires étrangères, rubrique politique étrangère de la France, le projet de Chaire UNESCO : http://www.diplomatie.gouv.fr/fr/enjeux-internationaux/ onu/commission-francaise-pour-l-unesco/reseaux-21483/reseau-des-chaires-unesco-unitwin/ article/le-projet-de-chaire-unesco [consulté le 29/08/2013].

63 Alain Chouraqui, op. cit. 
Force est de constater que le pari difficile a été gagné et que le mémorial des Milles, lieu souvenir, s'est transformé en un lieu de commémoration européen. L'objectif est tout à la fois de rendre hommage et d'éduquer pour l'avenir : la "mémoire révérence » est devenue la « mémoire référence ${ }^{64}$.

Cécile Denis

EA 4593 CLARE

Université Bordeaux Montaigne cecile.denis@etu.u-bordeaux-montaigne.fr

\section{Résumé}

Le mémorial du camp d'internement des Milles a été inauguré en septembre 2012 après soixante-dix ans d'oubli. Le présent article s'interroge sur les différents phénomènes mémoriels qui ont jalonné son histoire. En dépit de son caractère singulier, s'inscrit-il dans un paradigme mémoriel plus vaste, tel que l'histoire de Vichy ou la mémoire de l'exil allemand ? Quand cette mémoire s'est-elle réveillée et pour quelles raisons ? Qui en sont les instigateurs ? Quelles sont les manifestations organisées par le mémorial actuel et ses ambitions pour l'avenir ?

\section{Mots-clefs}

Camp d'internement, mémoire, Seconde guerre mondiale, exil, mémorial.

\section{Abstract}

The memorial of the Milles Internment Camp was inaugurated in September 2012 after 70 years of oblivion. This article focuses on the various memory phenomena that have punctuated its history. In spite of its singular nature, we can wonder if this memorial falls within a larger paradigm, such as the history of Vichy or the memory of the German exile. We can also ask ourselves when this need for memory arose, the reasons why it did so, and who are the people who triggered it? What kind of events are organized by the current memorial and what are its ambitions for the future?

\section{Keywords}

Internment Camp, Memory, Second World War, Exile, Memorial. 